\title{
Mesenchymal Stem Cell Therapy for Alzheimer's Disease
}

\author{
A. E. Hernández $(\mathbb{D})$ and E. García \\ Facultad de Ciencias de la Salud, Universidad Anáhuac México, Mexico \\ Correspondence should be addressed to E. García; edna.garcia@anahuac.mx
}

Received 15 June 2021; Accepted 9 August 2021; Published 1 September 2021

Academic Editor: Huseyin Sumer

Copyright (c) 2021 A. E. Hernández and E. García. This is an open access article distributed under the Creative Commons Attribution License, which permits unrestricted use, distribution, and reproduction in any medium, provided the original work is properly cited.

\begin{abstract}
Alzheimer's disease (AD) is a neurodegenerative disease responsible for $60-70 \%$ of the 50 million cases of dementia worldwide. It is characterized by neuronal cell death, shrinkage of brain tissue, and progressive cognitive, motor, and behavioral impairment, which often leads to death. Although current treatment has helped improve the patient's quality of life, it has not been able to alter the underlying disease pathology of AD. Studies have shown that mesenchymal stem cells (MSCs) - a group of multipotent stem cells-have the ability to stimulate neuroregeneration and inhibit disease progression. More recently, extracellular vesicles (EVs) from cytokine-preconditioned MSCs have also shown to induce immunomodulatory and neuroprotective effects in $\mathrm{AD}$ models. This review will aim to compile pertinent preclinical $\mathrm{AD}$ research on transgenic mice as well as clinical trials on MSC-based therapy from diverse sources.
\end{abstract}

\section{Introduction}

According to the World Health Organization, around 50 million individuals worldwide suffer from dementia [1]. This figure is expected to double every 20 years, until 2050 [2]. The most common form of dementia is Alzheimer's disease (AD) and may be responsible for $60-70 \%$ of the cases [1]. $\mathrm{AD}$ is a neurodegenerative disease characterized by the accumulation of extracellular amyloid plaques primarily composed of amyloid-beta (A $\beta$ ) peptides and intracellular neurofibrillary tangles (NFTs) of hyperphosphorylated tau protein [3]. Alois Alzheimer first described the disease in 1906 when he reported a 51-year-old woman with cognitive disturbances, delusions, disorientation, and other behavioral changes. $\mathrm{AD}$ is associated with neuronal cell death, shrinkage of brain tissue, and progressive cognitive, motor, and behavioral impairment, which often leads to death. As of today, disease-modifying treatments capable of altering the underlying disease pathology of $\mathrm{AD}$ are not available [4].

Mesenchymal stem cells (MSCs) are a group of multipotent stem cells capable of differentiating into nonmesenchymal lineages [5]. MSCs are considered a very promising approach to stimulate neuroregeneration due to their immunomodulatory properties and high biosafety but also because of their ability to synthetize neurotrophic and proangiogenic factors [6]. In $\mathrm{AD}$, stem cell therapies have attempted to replace neurons that have been impaired or lost. MSCbased therapies have shown potential to restore damaged neural tissue as well as slow disease progression. Although the specific therapeutic mechanism effect of stem cell transplantation is not known, MSC therapy has shown to be an alternative therapeutic approach for neurodegenerative diseases such as $\mathrm{AD}[5]$.

\section{Pathogenesis of AD and Current Treatment}

$\mathrm{AD}$ is believed to occur when extracellular senile plaques and intracellular NFTs of hyperphosphorylated tau protein accumulate in the brain. These pathological processes cause a series of downstream events that include neurodegeneration with neuronal and synaptic loss leading to macroscopic atrophy [7]. Although the pathophysiology of $\mathrm{AD}$ is still subject to debate, the predominant hypothesis known as the amyloid cascade hypothesis predicts that the accumulation and aggregation of $\mathrm{A} \beta$ provoke a pathological set of events that ultimately produce what we know as $\mathrm{AD}[3,4]$. The amyloid precursor protein (APP) is a transmembrane protein that plays an important role in neuronal development, signaling, 
and intracellular transport [8]. In the central nervous system (CNS), APP can undergo cleavage either by the nonamyloidogenic or amyloidogenic pathway. In the nonamyloidogenic pathway, APP is first cleaved by $\alpha$-secretase secreting the extracellular soluble peptide alpha $(\operatorname{sAPP} \alpha)$ and an amino acid fragment known $\mathrm{C} 83$, which is later cleaved by $\gamma$-secretase producing a fragment known as p3. On the other hand, in the amyloidogenic pathway, APP is first cleaved by $\beta$-secretase, secreting the extracellular soluble peptide beta ( $\operatorname{sAP} \beta)$ and an amino acid fragment called C99. $\gamma$-Secretase also participates in this pathway by cleaving C99, producing a 37-49 amino acid residue peptide known as $\mathrm{A} \beta$ [4]. The two main isoforms of $\mathrm{A} \beta$ are $\mathrm{A} \beta 40$ and $\mathrm{A} \beta 42$. The only difference between them is that $A \beta 42$ has two extra residues at the $C$ terminus. Most amyloid plaques in $\mathrm{AD}$ consist of predominantly $\mathrm{A} \beta 42$ [9]. The accumulation of $\mathrm{A} \beta$ monomers, as well as the decreased clearance, causes it to self-assemble and aggregate into oligomers and eventually into highly regular amyloid fibrils, generating plaques that lead to neurotoxicity and dementia [4]. Soluble $\mathrm{A} \beta$ and amyloid plaques are thought to induce a series of events like inflammation, oxidative stress, excitotoxicity, and hyperphosphorylation of tau. This is a protein known for stabilizing and assembling neuronal microtubules. In $\mathrm{AD}$, the hyperphosphorylation of tau promotes insoluble filamentous aggregates that are part of the intracellular NFTs characteristic of the disease [10]. Although the amyloid cascade hypothesis establishes that the hyperphosphorylation of tau is a downstream event of $\mathrm{A} \beta$ accumulation, it is possible that tau and $\mathrm{A} \beta$ act in parallel pathways causing $\mathrm{AD}$ and intensifying each other's toxic effects [2]. In addition, neuroinflammation has also demonstrated to play a key role in $\mathrm{AD}$ pathological process. Specifically, microglia have shown to be one of the main players orchestrating inflammation. In $\mathrm{AD}$, uncontrolled microglia activity aggravates tau pathology, stimulates the secretion of proinflammatory cytokines, and causes neuronal injury [11]. Lastly, mass neuronal and synaptic loss is also involved in the $\mathrm{AD}$ pathological process. Neurodegeneration affects the entorhinal cortex first, and then the subiculum and CA1 hippocampal subregion and basal forebrain networks. In the advanced stages of $\mathrm{AD}$, the temporal lobes are also affected, ultimately spreading through most cortical layers [12].

$\mathrm{AD}$ is commonly divided into familial $\mathrm{AD}$ (fAD) and sporadic $\mathrm{AD}(\mathrm{sAD})$. fAD is associated with early symptoms of onset and affects $<5 \%$ of all $\mathrm{AD}$ patients. This type of $\mathrm{AD}$ is caused by mutations in APP, presenilin 1 (PS1), and/or presenilin 2 (PS2) [13]. Presenilin 1 along with presenilin 2 is the catalytic components of the $\gamma$-secretase enzyme, which as we previously mentioned, cleaves APP into A $\beta$ s of varying lengths [14]. Thus, fAd-related mutations in presenilins alter the $\gamma$-secretase cleavage site, resulting in the generation of longer and more fibrillogenic $\mathrm{A} \beta$ [15]. On the other hand, $\mathrm{sAD}$, which is associated with late onset, represents nearly $95 \%$ of patients with $\mathrm{AD}$. sAD is caused by a combination of genetic and environmental risk factors without a documented familial history of AD. The apolipoprotein E4 (APOE4) and the triggering-receptor expressed on myeloid cells 2 (TREM2) have been considered the two major risk factors for sAD [13]. APOE genotypes have a sig- nificant impact on the deposition of $\mathrm{A} \beta$ to form senile plaques and cerebral amyloid angiopathy (CAA), two main hallmarks of amyloid pathology in AD [16]. Moreover, TREM2 mutations linked to AD generate a loss of TREM2 protein function, which is a receptor primarily expressed by microglia. This mutation affects the behavior of microglial cells, especially their response to $\mathrm{A} \beta$ plaques [17].

For both fAD and sAD, the current treatment includes two different types of pharmacological therapy. On one hand, cholinesterase inhibitors (ChEIs) such as donepezil, rivastigmine, and galantamine are prescribed for mild, moderate, or severe AD dementia [18]. ChEIs improve cognitive symptoms by inhibiting the biological activity of acetylcholinesterase and as a result increases acetylcholine levels in the synaptic cleft, a neurotransmitter used by cholinergic neurons which has been shown to improve the function of brain cells [19]. Response rates vary with approximately around one-third of individuals with $\mathrm{AD}$ showing no benefit at all and one-fifth having greater benefit. Similarly, about one-third of patients do not tolerate the ChEIs treatment approach because of side effects [20]. Meanwhile, in moderate to severe $\mathrm{AD}$ cases, patients are prescribed memantine, which inhibits the N-methyl-D-aspartate (NMDA) receptor and as a consequence may prevent neuron loss, as well as improve symptoms by restoring damaged neurons. In comparison, NMDA receptor antagonists have fewer side effects than ChEIs [18, 20]. Moreover, a nutraceutical, known as Huperzine A, is also used as an alternative treatment; however, it is not regulated by the Food and Drug Administration (FDA). It is important to note that although the current treatment has helped improve the quality of life of patients living with $\mathrm{AD}$ when used at the appropriate time, it has not changed the course or rate of decline of the disease [18]. It has a relatively small average overall effect over AD and is not capable of altering the course of the underlying neurodegenerative process [20].

\section{MSC as an Alternative Treatment}

Stem cells are undifferentiated cells that have the ability to self-renew, proliferate from a single cell, and differentiate into different types of cells and tissues. These cells can be grouped into 4 groups according to their origin: embryonic stem cells (ESCs), induced pluripotent stem cells (iPSCs), and fetal and adult stem cells. MSCs are an example of adult stem cells that can be obtained from various tissues including bone marrow, adipose tissue, bone, Wharton's jelly, umbilical cord blood, and peripheral blood [21]. According to the International Society for Cellular Therapy, MSCs are defined by expressing cluster differentiation (CD), CD90, CD73, CD105, and CD44 while not expressing CD45 and CD31 MSCs normal function in the body includes migrating to injury sites and taking part of a reparative process [22]. In comparison to other stem cells, MSCs differentiation potential is superior as they can differentiate into neuronal cells, osteocytes, chondrocytes, or adipocytes when stimulated with certain growth factors. In addition, MSCs also possess a low risk of differentiating into cancer cells and are infrequently immunogenic [23]. The latter is due to not expressing MHC 
class II and costimulatory molecules [24]. Moreover, MSCs also possess the ability to migrate to injury and hypoxia sites, boosting tissue repair, reducing apoptosis, and promoting angiogenesis [25].

In $\mathrm{AD}$ models, the reduction of $\mathrm{A} \beta$ plaques, $\beta$-secretase, and tau hyperphosphorylation as well as the reversal of microglial inflammation, and the stimulation of anti-inflammatory cytokines are among the mechanisms postulated to have a therapeutic effect in MSC therapy. Upregulating neuroprotection and downregulating proinflammatory cytokines have also been shown to have immunomodulatory and anti-inflammatory effects [26]. In addition, MSC excreted neurotrophic factors have been shown to be able to stimulate neurogenesis and synaptogenesis, alter immune cell response through overexpressing neuroprotective cytokines like IL-10, while reducing proinflammatory cytokines such as TNF- $\alpha$ and IL- $1 \beta$, increase the phagocytic activity of microglial cells, improve neovascularization, overcome cell death induced by $\mathrm{A} \beta$ and tau, reduce oxidative stress and apoptosis, alter autophagy pathways, and decrease plaque size [27]. Secreted MSC neurotrophic growth factors include glial cell-derived neurotrophic factor (GDNF), vascular endothelial growth factor (VEGF), brain-derived neurotrophic factor (BDNF), insulin-like growth factor 1(IGF-1), nerve growth factor (NGF), and fibroblast growth factor 2 (FGF2) [22, 27]. MSCs have also demonstrated to set off immune responses through microglia activation, which as a consequence sets off antiinflammatory response. They have also revealed to change microglia phenotype from classically activated to alternatively activated, causing a drop in proinflammatory cytokines and a rise of anti-inflammatory cytokines [28]. Moreover, MSCs also enhance nerve regeneration because of their ability to differentiate into Schwann cell-like cells, which are known for promoting regeneration. MSCs have also demonstrated to boost nerve regeneration via secretion of neurotrophic factors that induce axonal growth and stem cell differentiation into myelinating cell lines [25].

However, the specific mechanism effect of stem cell transplantation is still not known. Possible theories include direct cell replacement of injured neural cells, activation of endogenous stem cells, and secretion of neurotrophic and neuroprotective factors to increase cell survival [29]. In this regard, studies have suggested that the paracrine activity could be the key therapeutic mechanism due to research that indicated that secreted substances in MSC-derived conditioned media, rather than cell incorporation, were responsible for the considerable therapeutic effect in both in vitro and in vivo contexts [24].

\section{Preclinical Studies}

Many animal models of AD have been studied using different species, such as $C$. elegans, D. melanogaster, mouse, rat, and nonhuman primates [29]. However, the most used animal models are transgenic mice which overexpress or silence APP, PS1, and PS2 genes associated with fAD. However, these transgenic mice alone have not been able to replicate most of the pathophysiology of AD. With this in mind, a triple transgenic mouse model with modified APP, PS1, and
PS2 was generated. However, it is important to highlight that although a triple transgenic mouse is able to produce $A \beta$ plaques and NFTs, the progressive neuronal loss in the hippocampus and other regions of the human $\mathrm{AD}$ brain was not found $[30,31]$. On the other hand, no animal model is able to nearly resemble the mechanism and progression of the use of $s A D$, which is a significant restriction given that this type of $\mathrm{AD}$ is significantly more frequent than $\mathrm{fAD}$. As a result, many outcomes found in animal models are rarely seen in clinical studies [13]. The latest findings on MSC therapy in transgenic mouse models of $\mathrm{AD}$ will be presented in the next section. A summary is provided in Table 1.

The transplantation of Wharton's Jelly mesenchymal stem cells (WJ-MSCs) has been studied in AD mouse models. Lee et al. investigated the stereotactic administration of human WJ-MSCs and their secreted Agouti-related peptide (AgRP) into the hippocampi of 5XFAD mice which improved proteasome activity and reduced the accumulation of ubiquitin-conjugated proteins. The investigators suggest that either WJ-MSCs or AgRP may be used to delay the clinical progression of $\mathrm{AD}$ by improving proteasome activity and consequently lowering abnormal protein aggregation [32]. In another study, Xie et al. found that four weeks after transplantation, WJ-MSCs significantly enhanced spatial learning and mitigated memory decline in APP/PS1 mice. In addition, $\mathrm{A} \beta$ deposition and soluble $\mathrm{A} \beta$ levels were significantly reduced, while anti-inflammatory cytokine IL-10 significantly increased. Proinflammatory microglial activation and proinflammatory cytokines such as IL-1 $\beta$ and TNF $\alpha$ were also significantly reduced [33]. More recently, a group of researchers evaluated the difference between "prime" and "naïve" human WJ-MSCs. Prime human WJ-MSCs were previously exposed to an $\mathrm{AD}$ cell line via a coculture system and "naïve" human WJ-MSCs. Although both showed promising results in reducing cell death, ubiquitin conjugate levels, and A $\beta$ levels, "prime" human WJ-MSCs showed greater results. This suggests that exposure of human WJ-MSCs to an AD environment improves its therapeutic effects [34].

The transplantation of umbilical cord blood-derived mesenchymal stem cells (UCB-MSCs) has also showed promising results in $\mathrm{AD}$ mouse models. In a $5 \mathrm{xFAD}$ mouse AD model, human UCB-MS mitigated spatial learning and memory deterioration and alleviated the hyperphosphorylation of tau by GAL-3 secretion [35]. In the same regard, Kim et al. demonstrated that recurring intrathecal (I.T.) administration of human UCB-MSCs improves adult hippocampal neurogenesis and synaptic activity through growth differentiation factor-15 (GDF-15) secretion in an AD model [36]. Human UCB-MSCs have also demonstrated potential to attenuate $\mathrm{A} \beta 42$-induced synaptic dysfunction by regulating thrombospondin-1 (TSP-1) secretion [37]. In a study using Tg2576 mice, Cui et al. discovered that human UCB-MSC transplantation significantly mitigated cognitive deterioration of $\mathrm{AD}$ mice without altering $\mathrm{A} \beta$ levels in the hippocampus. They suggest that human UCB-MSCs may enhance cognitive deterioration by reducing oxidative stress and promoting hippocampal neurogenesis [38]. Moreover, Wang et al. demonstrated that resveratrol facilitates UCB-MSC 
TABLE 1: Most recent findings of MSCs in AD transgenic mice.

\begin{tabular}{|c|c|c|c|}
\hline Cell type & Model & Study design & Findings \\
\hline Human WJ-MSCs & 5XFAD & $\begin{array}{l}\text { Injection of WJ-MSCs or AgRP } \\
\text { directly into the left hippocampus } \\
\text { of 5XFAD mice. }\end{array}$ & $\begin{array}{l}\text { Improves proteasome activity by AgRP. } \\
\text { Reduces the accumulation of ubiquitin-conjugated } \\
\text { proteins [32]. }\end{array}$ \\
\hline Human WJ-MSCs & APP/PS1 & $\begin{array}{l}\text { Injection of WJ-MSCs into the } \\
\text { tail vein of APP/PS1 mice. }\end{array}$ & $\begin{array}{c}\text { Improves the spatial learning. } \\
\text { Mitigates memory decline. } \\
\text { Increases IL-10. } \\
\text { Reduces } \mathrm{A} \beta \text { deposition levels. } \\
\text { Reduces soluble A } \beta \text { levels. } \\
\text { Proinflammatory microglial activation. } \\
\text { Reduces IL-1 } \beta \text { and TNF } \alpha \text { levels [33]. }\end{array}$ \\
\hline Human WJ-MSCs & 5XFAD & $\begin{array}{l}\text { I.V.T. infusion of WJ-MSCs } \\
\text { (exposed to an AD cell line) into } \\
\text { 5XFAD mice. }\end{array}$ & $\begin{array}{l}\text { Reduces cell death } \\
\text { Reduces ubiquitin conjugate levels } \\
\text { Reduces } \mathrm{A} \beta \text { levels [34]. }\end{array}$ \\
\hline $\begin{array}{l}\text { Human UCB- } \\
\text { MSCs }\end{array}$ & 5XFAD & $\begin{array}{l}\text { Infusion of recombinant human } \\
\text { GAL-3 protein and UCB-MSCs } \\
\text { into } 5 \text { XFAD mice. }\end{array}$ & $\begin{array}{l}\text { Improves the spatial learning. } \\
\text { Improves memory impairment. } \\
\text { UCB-MSCs mitigate hyperphosphorylation of tau } \\
\text { through GAL-3 secretion [35]. }\end{array}$ \\
\hline
\end{tabular}

\begin{tabular}{lcc}
$\begin{array}{l}\text { Human UCB- } \\
\text { MSCs }\end{array}$ & APP/PS1 & $\begin{array}{c}\text { NSCs to identify paracrine factors } \\
\text { Repeated I.T. injections of } \\
\text { UCB-MSCs into APP/PS1 mice. }\end{array}$ \\
\hline & & $\begin{array}{c}\text { Coculture of UCB-MSCs with } \\
\text { primary hippocampal neurons }\end{array}$ \\
Human UCB- & under A $\beta 42$ peptide treatment \\
MSCs & to identify paracrine factors. \\
& Transplantation of hUCB-MSCs \\
& via I.C.V. route.
\end{tabular}

\begin{tabular}{|c|c|c|}
\hline $\begin{array}{l}\text { Human UCB- } \\
\text { MSCs }\end{array}$ & $\operatorname{Tg} 2576$ & $\begin{array}{c}\text { UCB-MSCs I.V. transplantation } \\
\text { into Tg2576 mice. }\end{array}$ \\
\hline
\end{tabular}

\begin{tabular}{|c|c|c|c|}
\hline $\begin{array}{l}\text { Human UCB- } \\
\text { MSCs }\end{array}$ & $\operatorname{Tg} 2576$ & $\begin{array}{l}\text { UCB-MSCs I.V. transplantation } \\
\text { combined with resveratrol } \\
\text { into Tg2576 mice. }\end{array}$ & $\begin{array}{l}\text { Better UCB-MSC engraftment in the hippocampus. } \\
\text { Improves learning and memory } \\
\text { Enhances neurogenesis } \\
\text { Alleviates neural apoptosis in the hippocampus [39]. }\end{array}$ \\
\hline $\begin{array}{l}\text { Human WJ-MSCs } \\
\text { and UCB-MSCs }\end{array}$ & 5XFAD & $\begin{array}{l}\text { Coculture of MSCs with SVZ-derived } \\
\text { NSCs from 5XFAD mice. }\end{array}$ & $\begin{array}{l}\text { Induces neuronal development and neurite outgrowth } \\
\qquad[40] .\end{array}$ \\
\hline Rat BM-MSCs & APP/PS1 & $\begin{array}{l}\text { I.C.V. injection of BM-MSCs } \\
\text { into APP/PS1 mice. }\end{array}$ & $\begin{array}{l}\text { Improves cognitive impairment by ameliorating astrocytic } \\
\text { inflammation as well as synaptogenesis by increasing the } \\
\text { expression of microRNA-146a in hippocampus [41]. }\end{array}$ \\
\hline $\begin{array}{l}\text { Human BM- } \\
\text { MSCs }\end{array}$ & APP/PS1 & $\begin{array}{l}\text { Tail I.V. injection of BM-MSCs } \\
\text { into APP/PS1 mice. }\end{array}$ & $\begin{array}{l}\text { Reduced levels of IL-1, IL-2, TNF- } \alpha \text {, and IFN- } \gamma \text {. } \\
\text { Regulates the expression of } \mathrm{A} \beta \text {-related genes [42]. }\end{array}$ \\
\hline Mouse BM-MSCs & $3 x \mathrm{Tg}-\mathrm{AD}$ & $\begin{array}{l}\text { Evaluation of I.V. injected BM-MSCs } \\
\text { using serial }[18 \mathrm{~F}] \text { florbetaben PET into } \\
\text { 3xTg-AD mice. }\end{array}$ & $\begin{array}{l}\text { The reduction of } \beta \text {-amyloid deposits during BMSCs } \\
\text { treatment could be confirmed by PET [ } 43] \text {. }\end{array}$ \\
\hline Mouse BM-MSCs & $3 \times \mathrm{Tg}-\mathrm{AD}$ & $\begin{array}{l}\text { Infusion of }{ }^{111} \text { In-labeled BM-MSCs via } \\
\text { I.V. administration into } 3 \times \text { Tg-AD mice. }\end{array}$ & $\begin{array}{c}\text { The number of BM-MSCs reaching the brain } \\
\text { is very small [44]. }\end{array}$ \\
\hline Murine BM-MSCs & APP/PS1 & $\begin{array}{l}\text { Injection of BM-MSCs into } \\
\text { APP/PS1 mice via the tail vein. }\end{array}$ & $\begin{array}{c}\text { Reduces pE3-A } \beta \text { plaque size. } \\
\text { Reduces gene expression of TNF- } \alpha \text {, IL-6, } \\
\text { MCP-1, and NGF. } \\
\text { Reduces microglial number and microglia size [45]. }\end{array}$ \\
\hline
\end{tabular}

Improves cognitive function Attenuates oxidative stress

Promotes cell proliferation and newborn cell survival Promotes neurons generating

Promotes hippocampal neurogenesis Increases expression of Sirt1, BDNF, and SYN [38].

Better UCB-MSC engraftment in the hippocampus. roves learning and memory improves endogenous hippocampal neur
and synaptic activity through CSF [36].

Mitigates A $\beta 42$-induced synaptic dysfunction by regulating TSP-1 release [37]. 
TABLE 1: Continued.

\begin{tabular}{|c|c|c|c|}
\hline Cell type & Model & Study design & Findings \\
\hline Murine BM-MSCs & APP/PS1 & $\begin{array}{l}\text { Single I.V. and repeated I.N. } \\
\text { administration of secretome } \\
\text { collected from MSCS exposed } \\
\text { in vitro to AD mouse brain } \\
\text { homogenates from APP/PS1 mouse. }\end{array}$ & $\begin{array}{c}\text { A single infusion: } \\
\text { Transient memory recovery } \\
\text { Improves the inflammatory phenotype of astrocytes. } \\
\text { Reduces brain amyloidosis and microglial activation. } \\
\text { Repeated infusions: } \\
\text { Sustains memory recovery } \\
\text { Reduces neuroinflammation } \\
\text { Decreases brain amyloidosis } \\
\text { Increases neuronal density in both cortex and } \\
\text { hippocampus } \\
\text { Diminishes hippocampal shrinkage [47]. }\end{array}$ \\
\hline
\end{tabular}

Improves the spatial learning and memory Mitigates amyloid plaques

$\begin{array}{lll}\text { Human MenSCs } & \text { APP/PS1 } & \begin{array}{l}\text { I.C. transplantation of MenSCs } \\ \text { into an APP/PS1 mice. }\end{array}\end{array}$

Reduces tau hyperphosphorylation

Increases $\mathrm{A} \beta$ degrading enzymes

Modulates a panel of proinflammatory cytokines associated with an altered microglial phenotype [48].

\begin{tabular}{|c|c|c|}
\hline Rat AD-MSCs & APP/PS1 & $\begin{array}{l}\text { Transplantation of AD-MSCs into } \\
\text { the hippocampi of APP/PS1 mice } \\
\text { with an automated infusion pump. }\end{array}$ \\
\hline
\end{tabular}

Reduces oxidative stress

Alleviates cognitive impairment

Promotes neurogenesis in the SGZ of the hippocampus

Increases the number of neuroblasts in the SVZ of the hippocampus [49].

\begin{tabular}{|c|c|c|c|}
\hline AM-MSCs & APP/PS1 & $\begin{array}{l}\text { Intrahippocampal transplantation } \\
\text { of AM-MSCs into APP/PS1 mice. }\end{array}$ & $\begin{array}{c}\text { Reduced amyloid- } \beta \text { peptide } \\
\text { (A } \beta) \text { deposition and rescued spatial learning and memory } \\
\text { Reduced amyloid- } \beta \text { peptide } \\
(\mathrm{A} \beta) \text { deposition and rescued spatial learning and memory } \\
\text { Improves the spatial learning and memory. } \\
\text { Reduces } \mathrm{A} \beta \text { deposition } \\
\text { Intensifies release of } \mathrm{A} \beta \text { degrading enzymes } \\
\text { Reduces microglia activation. } \\
\text { Increases hippocampal synaptic density and neurogenesis } \\
\text { mediated by BDNF [50]. }\end{array}$ \\
\hline MSC-EVs & $3 x \operatorname{Tg}$ & $\begin{array}{l}\text { Administration of EVs derived from } \\
\text { cytokine-preconditioned MSCs } \\
\text { through the I.N. route into 3xTg mice. }\end{array}$ & $\begin{array}{l}\text { Decrease microglia activation. } \\
\text { Increases dendritic spine density [11]. }\end{array}$ \\
\hline MSC-RVG-Exo & APP/PS1 mice & $\begin{array}{l}\text { Use of RVG peptide to target I.V. } \\
\text { infused MSC-Exo to the brain of } \\
\text { transgenic APP/PS1 mice. }\end{array}$ & $\begin{array}{l}\text { Improves cognitive function better than unmodified } \\
\text { exosomes. } \\
\text { Decrease plaque deposition and A } \beta \text { levels. } \\
\text { Reduces the activation of astrocytes. } \\
\text { Reduces the expression of proinflammatory mediators } \\
\text { such as TNF- } \alpha \text {, IL- } \beta \text {, and IL-6. } \\
\text { Raises the levels of IL-10, IL-4, and IL-13 [53]. }\end{array}$ \\
\hline \multicolumn{4}{|c|}{$\begin{array}{l}\text { AD-MSCs: adipose tissue-derived mesenchymal stem cells; AgRP: agouti-related peptide; AM-MSCs: amniotic mesenchymal stem cells; A } \beta \text { : amyloid-beta; } \\
\text { BDNF: brain-derived neurotrophic factor; BM-MSCs: bone marrow-derived mesenchymal stem cells; CSF: cerebrospinal fluid; EVs: extracellular vesicles; } \\
\text { GAL-3: galectin-3; I.C.: intracerebral; I.C.V.: intracerebroventricular; I.N.: intranasal; I.T.: intrathecal; I.V.: intravenous; I.V.T.: intraventricular; IFN- } \gamma \text { : } \\
\text { interferon-gamma; IL: interleukin; MCP-1: monocyte chemoattractant protein-1; MenSCs: menstrual blood-derived mesenchymal stem cells; MSC-EVs: } \\
\text { mesenchymal stem cell-derived extracellular vesicles; MSC-RVG-Exo: RVG-conjugated mesenchymal stem cell-derived; NGF: nerve growth factor; NSCs: } \\
\text { neural stem cells; pE3-A } \beta \text { : pyroglutamate modified form of amyloid-beta; PET: positron emission tomography; RVG: rabies viral glycoprotein; SGZ: } \\
\text { subgranular zone; Sirt1: sirtuin 1; SVZ: subventricular zone; SYN: synaptophysin; TNF- } \alpha \text { : tumor necrosis factor alpha; UCB-MSCs: umbilical cord blood- } \\
\text { derived mesenchymal stem cells; WJ-MSCs: Wharton's Jelly mesenchymal stem cells. }\end{array}$} \\
\hline
\end{tabular}

engraftment in the hippocampus and enhances its therapeutic effect [39]. Similarly, Park et al. studied the coculture of UCB-MSCs, WJ-MSCs, and SVZ-derived neural stem cells (NSCs) in an in vitro 5XFAD mouse model. Results demonstrated neuronal development and neurite outgrowth and an increased secretion of activin A. This suggests that both
MSCs and activin A may be used to improve neurogenesis for cortical regeneration to treat $\mathrm{AD}$ [40].

On the other hand, bone marrow-derived mesenchymal stem cells (BM-MSCs) have also been investigated. Intracerebroventricular (I.C.V.) BM-MSCs have shown potential to improve cognitive deterioration in $\mathrm{AD}$ model mice by 
mitigating astrocytic inflammation and synaptogenesis. Exosomal transfer of miR-146a-which accumulates in astrocytes-is suggested by Nakano et al. to be involved in the improvement of cognitive deterioration [41]. In another study, IL-1, IL-2, TNF- $\alpha$, and IFN- $\gamma$ levels were reduced after human BM-MSC treatment [42]. More recently, a group of researchers investigated the effects of BM-MSCs in $3 \mathrm{xTg} \mathrm{AD}$ mice using serial [18F] florbetaben positron emission tomography to evaluate the changes in $A \beta$ deposits after MSC therapy. Results showed that a reduction of $\mathrm{A} \beta$ deposits in AD mice during BMSC treatment could be confirmed by PET. This opens the possibility that noninvasive PET imaging may be used for the evaluation of MSC response in clinical applications [43]. In the same regard, Park et al. demonstrated that a small number of mouse BM-MSCs are able to reach the brain by intravenous (I.V.) infusion, and a greater distribution of BM-MSCs was found in the $3 \times \mathrm{Tg}-\mathrm{AD}$ mice compared to a control group. In addition, the results showed that brain uptake increased in the $\mathrm{AD}$ group, while the control group did not change significantly [44]. BM-MSC systemic administration in APP/PS1 mouse has also shown the potential to reduce pyroglutamate-modified amyloid-beta $(\mathrm{pE} 3-\mathrm{A} \beta)$ plaque size, a potential biomarker for $\mathrm{A} \beta$ plaque pathology $[45,46]$. The systemic administration of secretomes isolated from mouse BM-MSCs exposed to AD mouse brain homogenates has also been studied. Santamaria et al. demonstrated that the secretome derived from AD-conditioned MSCs can fully replicate the neuroreparative effects previously associated with MSC direct transplantation. Repeated intranasal (I.N.) infusions of the secretome of APP/PS1 fully restored mouse memory and improved the neuropathology in advanced stages of the disease. Preconditioning BM-MSCs to an AD environment showed to be crucial to induce the therapeutic activity of the secretome [47].

The therapeutic effects of adipose tissue, menstrual blood, and amniotic fluid mesenchymal stem cells have also been studied. Zhao et al. studied menstrual blood-derived mesenchymal stem cells (MenSCs) that showed that intracerebral (IC) injection of them improved $\mathrm{A} \beta$ plaques, reduced tau hyperphosphorylation, and enhanced the spatial learning and memory of APP/PS1 mice. They also demonstrated that MenSCs were able to increment various $\mathrm{A} \beta$ degrading enzymes and decrease several proinflammatory cytokines such as IL- $1 \beta$ and TNF- $\alpha$, which as we previously mentioned are associated with an altered microglial phenotype [48]. On the other hand, adipose tissue-derived mesenchymal stem cells (AD-MSCs) showed to improve endogenous neurogenesis in both the subgranular and subventricular zones, reduce oxidative stress, and mitigate cognitive deterioration in APP/PS1 mice [49]. Similarly, human amniotic mesenchymal stem cell (AM-MSC) intrahippocampal transplantation significantly diminished $\mathrm{A} \beta$ deposition and improved the spatial learning and memory deficits in APP/PS1 mice. Zheng et al. suggest that improved cognition may be associated with an increment in hippocampal synaptic density and neurogenesis that is regulated by BDNF [50].

Extracellular vesicles (EVs) from cytokine-preconditioned MSCS are also being investigated as a therapeutic approach for
$\mathrm{AD}$ due to MSCs reporting paracrine activity. EVs such as exosomes and microvesicles are lipid bilayer vesicles secreted by cells into the extracellular space $[11,51]$. Lourdo et al. found that mesenchymal stem cell-derived extracellular vesicles (MCS-EVs) regulate the microglia activation and decreases dendritic spine loss in a triple-transgenic $3 \mathrm{xTg}$ mouse [11]. Another study showed that I.V. injected exosomes accumulate mainly in the spleen and liver and not in the brain [52]. Therefore, Cui et al. proposed surface-modified MSCEVs that target the cortex and hippocampus of AD mice by using a specific rabies viral glycoprotein (RVG) peptide to prevent memory deficits in AD. Results showed that RVGconjugated mesenchymal stem cell-derived exosomes (MSCRVG-Exo) improved memory deficits, decreased plaque deposition and $\mathrm{A} \beta$ levels, and normalized levels of inflammatory cytokines compared to unmodified exosomes [53].

\section{Clinical Studies}

Many clinical trials have been conducted to evaluate the role of MSCS derived from various sources in patients with AD. It is important to note that only a few have been officially completed and have published results. A summary of the clinical trials registered on ClinicalTrials.gov evaluating MSCs for AD treatment can be found in Table 2.

5.1. Completed. A phase 1 clinical trial evaluated a single stereotactic brain infusion of human UCB-MSCs in patients with mild-to-moderate AD. 3 subjects received a single low dose infusion $\left(3.0 \times 10^{6}\right.$ cells $\left./ 60 \mu \mathrm{L}\right)$ while 6 subjects received a high dose infusion $\left(6.0 \times 10^{6}\right.$ cells $\left./ 60 \mu \mathrm{L}\right)$. Results showed that a single injection of human UCB-MSCs was safe and well tolerated and did not cause serious adverse effects during the two-year follow-up. However, it failed to produce the same therapeutic effect as seen in animal studies. Kim et al. speculate that the discrepancy between animal and human studies may be related to the Pittsburgh compound $\mathrm{B}$ positron emission tomography (PiB-PET), which may not be sensitive enough to detect soluble amyloid or diffuse amyloid plaques in comparison with immunohistochemical staining, enzyme-linked immunosorbent assay, and Western blot used in preclinical studies, which are capable of detecting soluble and insoluble amyloid as well as different types of amyloid plaques. Another reason as to why the human brain did not replicate findings from the animal studies may be due to their AD microenvironment differences. Similarly, preclinical studies rely on xenogeneic transplantation where as human clinical trials rely on allogenic transplantation. Some limitations of this clinical study include the lack of a control group and the small number of participants [54-56].

A phase $1 / 2$ a trial evaluated the safety, dose-limiting toxicity, and exploratory efficacy of 3 repeated intraventricular administrations (I.V.T.) of NEUROSTEM ${ }^{\circledR}$ (human UCBMSCs) versus placebo via an Ommaya reservoir at 4 week intervals in subjects with $\mathrm{AD}$ was completed in December 2019. The study was divided into two stages, stage 1 consisted of a dose escalation in which 3 subjects received a low dose $\left(1 \times 10^{7}\right.$ cells $\left./ 2 \mathrm{~mL}\right)$ and 6 subjects a high dose $\left(3 \times 10^{7}\right.$ cells $\left./ 2 \mathrm{~mL}\right)$. On the other hand, stage 2 , made up 


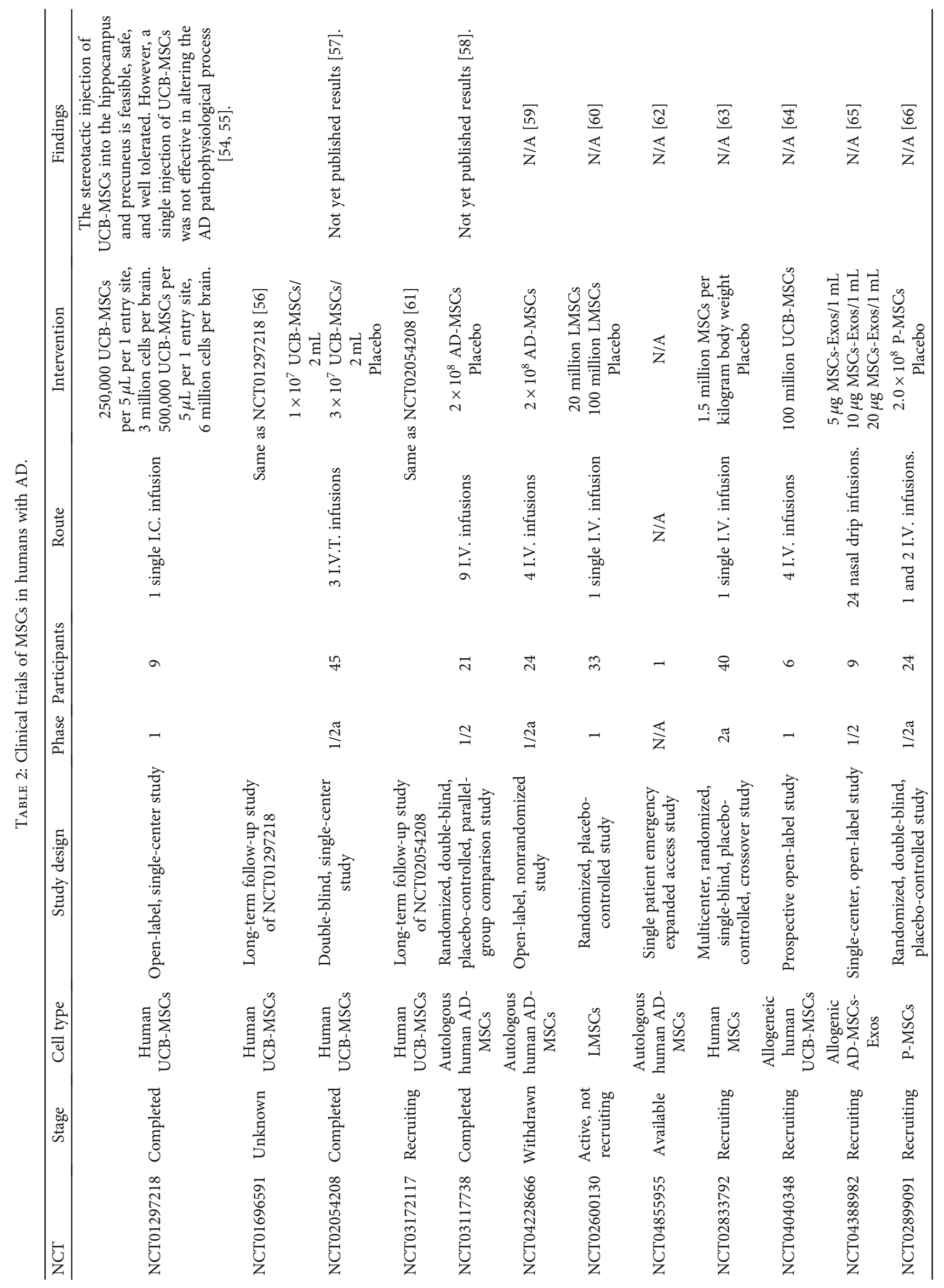




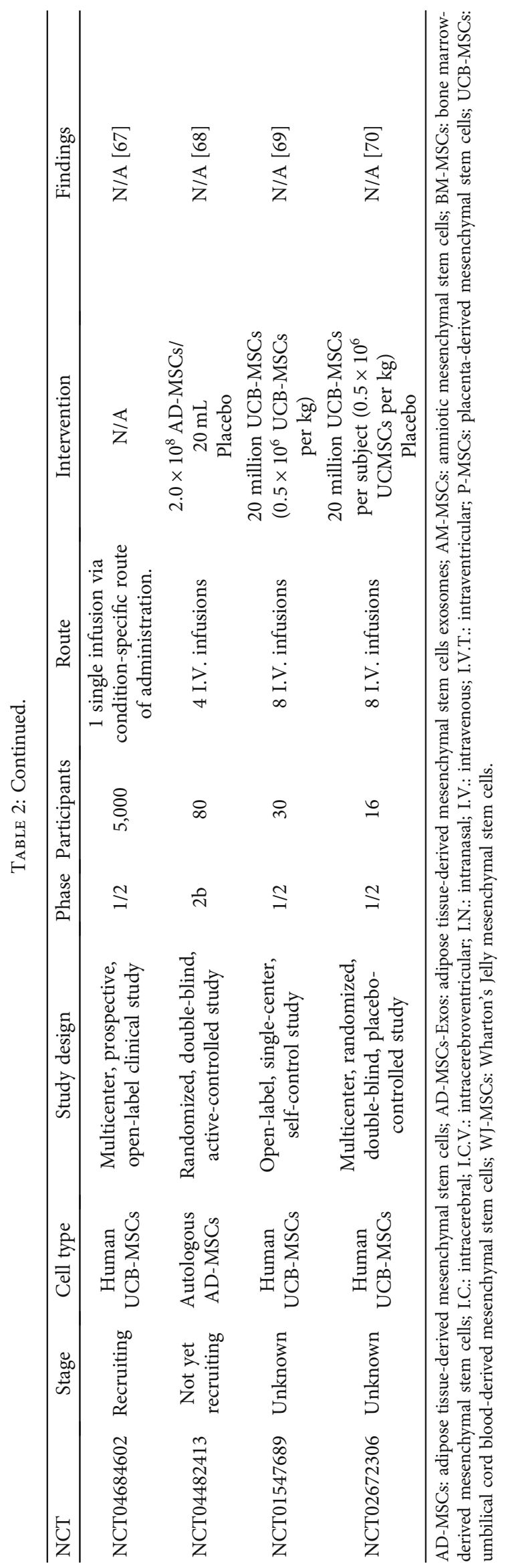


by a randomized and multiple-dose cohort parallel design, consisted of 36 individuals, in which 24 subjects received a high dose with the concentration previously mentioned and 12 received placebo. All groups received 3 repeated I.V.T. infusions via an Ommaya Reservoir at 4 week intervals. The study has yet to publish the results [57].

A phase $1 / 2$ randomized, double-blind, placebocontrolled study was completed on 2019.21 subjects were randomly assigned into the AstroStem (autologous human ADMSCs) and placebo control groups in a 1:1 ratio. Both groups received 9 repeated I.V. infusions at 2 week intervals, however, only 7 subjects completed the AstroStem infusions and 5 the placebo. Results showed 3 serious adverse effects in the AstroStem group including diarrhea, oesophageal, squamous cell carcinoma stage IV, and pulmonary embolism. Other reported adverse effects were open-angle glaucoma, dysphagia, fatigue, infusion site reaction, fall, dehydration, abnormal loss of weight, syncope, and ecchymosis. The changes in ADAS-Cog score from baseline to week 30 in the AstroStem group were 5.9 (6.8) and 3.0 (5.4) in the control group [58].

5.2. Ongoing. A phase $1 / 2 \mathrm{a}$, open-label, which is currently an ongoing schedule to end in February 2022, was designed to evaluate the safety profile of $4 \mathrm{I}$.V. infusions of autologous adipose-derived mesenchymal stem cells (HB-adMSCs) in 24 subjects as a possible treatment for AD. 4 I.V. infusions were administered on weeks $0,2,6$, and 8 at a dose of $2 \times$ $10^{8}$ total HB-adMSC cells. The main purpose was to evaluate the adverse effects of their treatment as well as the ability of $\mathrm{HB}$-adMSCs to alter $\mathrm{AD}$-related inflammation via measuring the levels of tumor necrosis factor-alpha (TNF-a), interleukin-1 (IL-1), interleukin-6 (IL-6), C-Reactive Protein $(\mathrm{CRP})$, and markers associated with amyloid deposition, $\mathrm{A} \beta 40$, and $\mathrm{A} \beta 42$. Participants will also be evaluated for cognitive deficits determined by changes in standard values of MMSE, ADCS-ADL, Alzheimer's disease Related Quality of Life (ADRQL), Altoida Neuro Motor Index (NMI) for Digital Biomarkers, and Clinical Dementia Rating Questionnaire (CDR) [59].

A phase 1 placebo-controlled clinical trial designed to evaluate the safety and efficacy of Longeveron Mesenchymal Stem Cells (LMSCs) in individuals with AD is currently ongoing.

A single peripheral I.V. infusion was administered in 25 subjects. Group 1 (10 individuals) received 20 million LMSCs (low-dose), group 2 (10 individuals) received 100 million LMSCs (high-dose), and group 3 (5 individuals) received placebo. To evaluate the safety of the infusion, the incidence of any treatment-emergent serious adverse events was assessed within the first 30 days after treatment administration. Preliminary efficacy was determined by changes in standard values for ADAS-Cog, MMSE, NPI, GDS, University of Pennsylvania Smell Identification Test (UPSIT), ADCS-ADL, Quality of Life-Alzheimer's Disease (QOL$\mathrm{AD})$, blood inflammatory and AD biomarkers, cerebrospinal fluid inflammatory biomarkers, CSF biomarkers of AD, and brain volumetry calculated using MRI at baseline, 2, 4, 13, 26,39 , and 52 weeks' postinfusion [60].
5.3. Challenges. Despite all favorable findings found in MSC$\mathrm{AD}$ therapy, this treatment has several limitations. To begin, no animal model is able to fully replicate the entire pathophysiology of the disease. Similarly, most transgenic mice utilized in preclinical studies rely on the expression of fAD mutations, the least frequent type of $\mathrm{AD}$, while most clinical trials consist of patients with SAD. This complicates the translation of fundamental research findings into clinical investigations. The lack of an effective model that can replicate the entire pathophysiology of the disease is one of the biggest setbacks in the search for an effective AD treatment [31].

Human UCB-MSCs are the most often employed MSCs in clinical studies. However, further research is required to determine if this source is truly the most effective. Correspondingly, the number of injections and delivery routes must also be optimized [71]. In this regard, of the only three completed clinical trials, they all used a different delivery route including I.V., I.V.T., and I.C. The most effective and noninvasive route must be used as repeated infusions will be needed considering that NCT01297218 showed that a single infusion of MSCs was not enough to alter the AD pathophysiological process $[11,54]$. Furthermore, further research and appropriate assessment techniques are required to prove the absence of adverse effects from MSC treatment on a long-term basis.

In regard to the administration of MSC-EVs, although preclinical studies have shown promising potential therapeutic effects, it has yet to be studied in patients with AD. As of May 31, 2021, only one clinical trial which is currently recruiting participants is set to study the safety and efficacy of exosomes for AD treatment. However, as suggested by Salem et al., some limitations including EVs heterogeneity, isolation, and production techniques need to be assessed first [28].

\section{Conclusions}

Many preclinical trials evaluating MSC treatment in transgenic mouse models of $\mathrm{AD}$ have yielded promising therapeutic outcomes. However, this has not been the case with human clinical trials. Although MSC therapy has demonstrated to be safe and tolerable in a single clinical study with published findings, the therapeutic benefit was not replicated. The frequency of unsuccessful clinical trials might be lowered by developing a successful AD model, notably the one for sAD, which accounts for the vast majority of $\mathrm{AD}$ patients. This will allow for a more efficient transition from basic research to clinical trials. Nonetheless, we believe that MSC therapy has a promising future and that its paracrine function, in particular, must be harnessed to establish a successful treatment for $\mathrm{AD}$.

\section{Data Availability}

No datasets were generated during the current study.

\section{Conflicts of Interest}

The authors declare that there is no conflict of interest regarding the publication of this paper. 


\section{Acknowledgments}

This review was funded by Fondo de Investigación de la Universidad Anáhuac, México.

\section{References}

[1] Dementia, "Who.int," 2021, https://www.who.int/news-room/ fact-sheets/detail/dementia/.

[2] P. Scheltens, K. Blennow, M. Breteler et al., "Alzheimer's disease," The Lancet, vol. 388, no. 10043, pp. 505-517, 2016.

[3] I. Lauritzen, R. Pardossi-Piquard, C. Bauer et al., "The -secretase-derived C-terminal fragment of APP, C99, but not A , is a key contributor to early intraneuronal lesions in tripletransgenic mouse hippocampus," Journal of Neuroscience, vol. 32, no. 46, pp. 16243-16255, 2012.

[4] J. Soria Lopez, H. González, and G. Léger, "Alzheimer's disease," in Handbook of Clinical Neurology, pp. 231-255, Geriatric Neurology. ST. DeKosky and S. Asthana Editors. Elsevier, 2019.

[5] F. Chakari-Khiavi, S. Dolati, A. Chakari-Khiavi et al., "Prospects for the application of mesenchymal stem cells in Alzheimer's disease treatment," Life Sciences, vol. 231, article 116564, 2019.

[6] Y. Mukhamedshina, O. Gracheva, D. Mukhutdinova, Y. Chelyshev, and A. Rizvanov, "Mesenchymal stem cells and the neuronal microenvironment in the area of spinal cord injury," Neural Regeneration Research, vol. 14, no. 2, article 244778, pp. 227-237, 2019.

[7] C. Lane, J. Hardy, and J. Schott, “Alzheimer's disease," European Journal of Neurology, vol. 25, no. 1, pp. 59-70, 2018.

[8] G. Chen, T. Xu, Y. Yan et al., "Amyloid beta: structure, biology and structure-based therapeutic development," Acta Pharmacologica Sinica, vol. 38, no. 9, pp. 1205-1235, 2017.

[9] L. Gu and Z. Guo, "Alzheimer's A $\beta 42$ and A $\beta 40$ peptides form interlaced amyloid fibrils," Journal of Neurochemistry, vol. 126, no. 3, pp. 305-311, 2013.

[10] D. Barragán Martínez, M. García Soldevilla, A. Parra Santiago, and J. Tejeiro Martínez, "Alzheimer's disease," Medicine - Programa de Formación Médica Continuada Acreditado, vol. 12, no. 74, pp. 4338-4346, 2019.

[11] M. Losurdo, M. Pedrazzoli, C. D'Agostino et al., "Intranasal delivery of mesenchymal stem cell-derived extracellular vesicles exerts immunomodulatory and neuroprotective effects in a 3xTg model of Alzheimer's disease," STEM CELLS Translational Medicine, vol. 9, no. 9, pp. 1068-1084, 2020.

[12] T. Duncan and M. Valenzuela, "Alzheimer's disease, dementia, and stem cell therapy," Stem Cell Research \& Therapy, vol. 8, no. 1, p. 111, 2017.

[13] L. Zhang, C. Chen, M. Mak et al., "Advance of sporadic Alzheimer's disease animal models," Medicinal Research Reviews, vol. 40, no. 1, pp. 431-458, 2020.

[14] B. De Strooper, "Loss-of-function presenilin mutations in Alzheimer disease," EMBO reports, vol. 8, no. 2, pp. 141-146, 2007.

[15] M. Kabir, M. Uddin, J. Setu, G. Ashraf, M. Bin-Jumah, and M. Abdel-Daim, "Exploring the role of PSEN mutations in the pathogenesis of Alzheimer's disease," Neurotoxicity Research, vol. 38, no. 4, pp. 833-849, 2020.

[16] C. Liu, C. C. Liu, T. Kanekiyo, H. Xu, and G. Bu, "Apolipoprotein E and Alzheimer disease: risk, mechanisms and therapy," Nature Reviews Neurology, vol. 9, no. 2, pp. 106-118, 2013.
[17] S. Carmona, K. Zahs, E. Wu, K. Dakin, J. Bras, and R. Guerreiro, "The role of TREM2 in Alzheimer's disease and other neurodegenerative disorders," The Lancet Neurology, vol. 17, no. 8, pp. 721-730, 2018.

[18] J. Weller and A. Budson, "Current understanding of Alzheimer's disease diagnosis and treatment," F1000Research, vol. 7, article 1161, 2018.

[19] K. Sharma, "Cholinesterase inhibitors as Alzheimer's therapeutics (Review)," Molecular Medicine Reports, vol. 20, no. 2, pp. 1479-1487, 2019.

[20] R. Briggs, S. Kennelly, and D. O'Neill, "Drug treatments in Alzheimer's disease," Clinical Medicine, vol. 16, no. 3, pp. 247253, 2016.

[21] G. Kolios and Y. Moodley, "Introduction to stem cells and regenerative medicine," Respiration, vol. 85 , no. 1, pp. 3-10, 2013.

[22] N. P. Staff, D. Jones, and W. Singer, "Mesenchymal stromal cell therapies for neurodegenerative diseases," Mayo Clinic Proceedings, vol. 94, no. 5, pp. 892-905, 2019.

[23] J. Kim, Y. Lee, S. Lee, K. Kim, M. Song, and J. Lee, "Mesenchymal stem cell therapy and Alzheimer's disease: current status and future perspectives," Journal of Alzheimer's Disease, vol. 77, no. 1, pp. 1-14, 2020.

[24] L. Liew, T. Katsuda, L. Gailhouste, H. Nakagama, and T. Ochiya, "Mesenchymal stem cell-derived extracellular vesicles: a glimmer of hope in treating Alzheimer's disease," International Immunology, vol. 29, no. 1, pp. 11-19, 2017.

[25] D. Cooney, E. Wimmers, Z. Ibrahim et al., "Mesenchymal stem cells enhance nerve regeneration in a rat sciatic nerve repair and hindlimb transplant model," Scientific Reports, vol. 6 , no. $1,2016$.

[26] X. Liu, L. Yang, and L. Zhao, "Stem cell therapy for Alzheimer's disease," World Journal of Stem Cells, vol. 12, no. 8, pp. 787802, 2020.

[27] M. Alipour, S. Nabavi, L. Arab et al., "Stem cell therapy in Alzheimer's disease: possible benefits and limiting drawbacks," Molecular Biology Reports, vol. 46, no. 1, pp. 1425-1446, 2019.

[28] H. Salem, G. Colpo, and A. Teixeira, "Stem cells in Alzheimer's disease: current standing and future challenges," Advances in Experimental Medicine and Biology, vol. 1079, pp. 93-102, 2018.

[29] F. Han, J. Bi, L. Qiao, and O. Arancio, "Stem cell therapy for Alzheimer's disease," Advances in Experimental Medicine and Biology, vol. 1266, pp. 39-55, 2020.

[30] F. Han, W. Wang, and C. Chen, "Research progress in animal models and stem cell therapy for Alzheimer\&rsquo;s disease," Journal of Neurorestoratology, vol. 2015, no. 3, pp. 11-22, 2014.

[31] E. Drummond and T. Wisniewski, "Alzheimer's disease: experimental models and reality," Acta Neuropathologica, vol. 133, no. 2, article 1662, pp. 155-175, 2017.

[32] N. Lee, S. Park, S. Kwon et al., "Agouti Related Peptide Secreted Via Human Mesenchymal Stem Cells Upregulates Proteasome Activity in an Alzheimer's Disease Model," Scientific Reports, vol. 7, no. 1, 2017.

[33] Z. Xie, Z. Liu, X. Zhang et al., "Wharton's Jelly-derived mesenchymal stem cells alleviate memory deficits and reduce amyloid- $\beta$ deposition in an APP/PS1 transgenic mouse model," Clinical and Experimental Medicine, vol. 16, no. 1, pp. 89-98, 2016.

[34] S. Park, H. Kim, S. Kwon et al., "Exposure of mesenchymal stem cells to an Alzheimer's disease environment enhances 
therapeutic effects," Stem Cells International, vol. 2021, Article ID 6660186, 14 pages, 2021.

[35] H. Lim, D. Lee, W. Choi, S. Choi, W. Oh, and D. Kim, “Galectin-3 secreted by human umbilical cord blood-derived mesenchymal stem cells reduces aberrant tau phosphorylation in an Alzheimer disease model," Stem Cells International, vol. 2020, Article ID 8878412, 14 pages, 2020.

[36] D. Kim, D. Lee, E. Chang et al., "GDF-15 secreted from human umbilical cord blood mesenchymal stem cells delivered through the cerebrospinal fluid promotes hippocampal neurogenesis and synaptic activity in an Alzheimer's disease model," Stem Cells and Development, vol. 24, no. 20, pp. 2378-2390, 2015.

[37] D. Kim, H. Lim, D. Lee et al., “Thrombospondin-1 secreted by human umbilical cord blood-derived mesenchymal stem cells rescues neurons from synaptic dysfunction in Alzheimer's disease model," Scientific Reports, vol. 8, no. 1, article 18542, p. 354, 2018.

[38] Y. Cui, S. Ma, C. Zhang et al., "Human umbilical cord mesenchymal stem cells transplantation improves cognitive function in Alzheimer's disease mice by decreasing oxidative stress and promoting hippocampal neurogenesis," Behavioural Brain Research, vol. 320, pp. 291-301, 2017.

[39] X. Wang, S. Ma, B. Yang et al., "Resveratrol promotes hUCMSCs engraftment and neural repair in a mouse model of Alzheimer's disease," Behavioural Brain Research, vol. 339, pp. 297-304, 2018.

[40] S. Park, J. Lee, E. Chang et al., “Activin A secreted by human mesenchymal stem cells induces neuronal development and neurite outgrowth in an in vitro model of Alzheimer's disease: neurogenesis induced by MSCs via activin A," Archives of Pharmacal Research, vol. 39, no. 8, pp. 1171-1179, 2016.

[41] M. Nakano, K. Kubota, E. Kobayashi et al., "Bone marrowderived mesenchymal stem cells improve cognitive impairment in an Alzheimer's disease model by increasing the expression of microRNA-146a in hippocampus," Scientific Reports, vol. 10, no. 1, article 10772, 2020.

[42] Y. Wei, Z. Xie, J. Bi, and Z. Zhu, "Anti-inflammatory effects of bone marrow mesenchymal stem cells on mice with Alzheimer's disease," Experimental and Therapeutic Medicine, vol. 16, no. 6, pp. 5015-5020, 2018.

[43] B. Park, J. Kim, T. Lim et al., "Therapeutic effect of mesenchymal stem cells in an animal model of Alzheimer's disease evaluated by $\beta$-amyloid positron emission tomography imaging," Australian \& New Zealand Journal of Psychiatry, vol. 54, no. 9, pp. 883-891, 2020.

[44] B. Park, T. Lim, J. Yoon, and Y. An, "In vivo tracking of intravenously injected mesenchymal stem cells in an Alzheimer's animal model," Cell Transplantation, vol. 27, no. 8, pp. 1203-1209, 2018.

[45] Y. Naaldijk, C. Jäger, C. Fabian et al., "Effect of systemic transplantation of bone marrow-derived mesenchymal stem cells on neuropathology markers in APP/PS1 Alzheimer mice," Neuropathology and Applied Neurobiology, vol. 43, no. 4, pp. 299-314, 2017.

[46] P. Wang, K. Lin, H. Liu et al., "Plasma pyroglutamate-modified amyloid beta differentiates amyloid pathology," Alzheimer's \& Dementia: Diagnosis, Assessment \& Disease Monitoring, vol. 12, no. 1, article e12029, 2020.

[47] G. Santamaria, E. Brandi, P. Vitola et al., "Intranasal delivery of mesenchymal stem cell secretome repairs the brain of Alz- heimer's mice," Cell Death \& Differentiation, vol. 28, no. 1, pp. 203-218, 2021.

[48] Y. Zhao, X. Chen, Y. Wu, Y. Wang, Y. Li, and C. Xiang, "Transplantation of human menstrual blood-derived mesenchymal stem cells alleviates Alzheimer's disease-like pathology in APP/PS1 transgenic mice," Frontiers in Molecular Neuroscience, vol. 11, 2018.

[49] Y. Gong, Y. Yan, T. Ma, K. Gong, Q. Ao, and X. Zhang, "Adipose-derived mesenchymal stem cell transplantation promotes adult neurogenesis in the brains of Alzheimer's disease mice," Neural Regeneration Research, vol. 9, no. 8, article 131596, pp. 798-805, 2014.

[50] X. Zheng, Q. Wan, C. Zheng et al., “Amniotic mesenchymal stem cells decrease $\mathrm{A} \beta$ deposition and improve memory in APP/PS1 transgenic mice," Neurochemical Research, vol. 42, no. 8, pp. 2191-2207, 2017.

[51] L. Doyle and M. Wang, "Overview of extracellular vesicles, their origin, composition, purpose, and methods for exosome isolation and analysis," Cell, vol. 8, no. 7, p. 727, 2019.

[52] C. Lai, O. Mardini, M. Ericsson et al., "Dynamic biodistribution of extracellular vesicles in vivo using a multimodal imaging reporter," ACS Nano, vol. 8, no. 1, pp. 483-494, 2014.

[53] G. Cui, H. Guo, H. Li et al., "RVG-modified exosomes derived from mesenchymal stem cells rescue memory deficits by regulating inflammatory responses in a mouse model of Alzheimer's disease," Immunity \& Ageing, vol. 16, no. 1, 2019.

[54] H. Kim, S. Seo, J. Chang et al., "Stereotactic brain injection of human umbilical cord blood mesenchymal stem cells in patients with Alzheimer's disease dementia: a phase 1 clinical trial," Alzheimer's \& Dementia: Translational Research \& Clinical Interventions, vol. 1, no. 2, pp. 95-102, 2015.

[55] "The safety and the efficacy evaluation of NEUROSTEM ${ }^{\circledR}$-AD in patients with Alzheimer's disease," 2021, https:// clinicaltrials.gov/ct2/show/NCT01297218.

[56] "The long-term safety and efficacy follow-up study of subjects who completed the Phase I Clinical Trial of Neurostem ${ }^{\circledR}$-AD," 2021, https://clinicaltrials.gov/ct2/show/NCT01696591.

[57] "Safety and exploratory efficacy study of NEUROSTEM ${ }^{\circledR}$ versus placebo in patients with Alzheimer's disease," 2021, https:// clinicaltrials.gov/ct2/show/NCT02054208.

[58] "A study to evaluate the safety and efficacy of AstroStem in treatment of Alzheimer's disease," 2021, https://clinicaltrials .gov/ct2/show/NCT03117738.

[59] "A clinical trial to determine the safety and efficacy of hope biosciences autologous mesenchymal stem cell therapy (HBadMSCs) for the treatment of Alzheimer's disease," 2021, https://clinicaltrials.gov/ct2/show/NCT04228666.

[60] "Allogeneic human mesenchymal stem cell infusion versus placebo in patients with Alzheimer's disease," 2021, https:// clinicaltrials.gov/ct2/show/NCT02600130.

[61] "Follow-up study of safety and efficacy in subjects who completed NEUROSTEM ${ }^{\circledR}$ Phase-I/IIa Clinical Trial," 2021, https://clinicaltrials.gov/ct2/show/NCT03172117.

[62] "Autologous human adipose-derived mesenchymal stem cells in Alzheimer's disease," 2021, https://clinicaltrials.gov/ct2/ show/NCT04855955?term $=$ Mesenchymal+stem + cell\&cond $=$ Alzheimer+Disease\&draw $=3 \&$ rank $=3$.

[63] "Allogeneic human mesenchymal stem cells for Alzheimer's disease," 2021, https://clinicaltrials.gov/ct2/show/NCT02833792.

[64] "Alzheimer's disease stem cells multiple infusions," 2021, https://clinicaltrials.gov/ct2/show/NCT04040348. 
[65] "The safety and the efficacy evaluation of allogenic adipose MSC-Exos in patients with Alzheimer's disease," 2021, https:// clinicaltrials.gov/ct2/show/NCT04388982.

[66] "Evaluation of the safety and potential therapeutic effects after intravenous transplantation of CB-AC-02 in patients with Alzheimer's disease," 2021, https://clinicaltrials.gov/ct2/show/ NCT02899091.

[67] "Mesenchymal stem cells for the treatment of various chronic and acute conditions," 2021, https://clinicaltrials.gov/ct2/ show/NCT04684602.

[68] "Study to evaluate the safety and efficacy of AstroStem in treatment of Alzheimer's disease," 2021, https://clinicaltrials.gov/ ct2/show/NCT04482413.

[69] "Safety and efficiency of umbilical cord-derived mesenchymal stem cells(UC-MSC) in patients with Alzheimer's disease (SEMAD)," 2021, https://clinicaltrials.gov/ct2/show/ NCT01547689.

[70] "Safety and exploratory efficacy study of UCMSCs in patients with Alzheimer's disease (SEESUPAD)," 2021, https:// clinicaltrials.gov/ct2/show/NCT02672306.

[71] S. Wang, C. Lee, and H. Lim, "Stem cell therapies for Alzheimer's disease," Current Opinion in Psychiatry, vol. 32, no. 2, pp. 105-116, 2019. 